\title{
FPGA technology and its application in FCL control design
}

\author{
XiaoBao Zhang \\ JiXi,AnHui Pumped Storage Limited Company \\ Email:344719616@qq,com
}

\begin{abstract}
Keywords: Fault Current Limiter(FCL);Short Circuit; Fast Detection; Field-Programmable Gate Array (FPGA)
\end{abstract}

Abstract. This paper proposed a fault fast detection criterion based on the change of voltage on the resonant device after a fault occurs, this proposition is based on a series analysis of the resonance voltage waveform on the acceptor resonance fault current limiter (FCL) in the OMIB system. Meanwhile, possible solutions to prevent malfunction from the turbulences caused by line breakage and the close and open operation of circuit breaker, in which operating is undesired. With help of the power systems computer aided design (PSCAD) software, simulation of the system under different fault is done. Verify that the criterion can meet the speed requirements of the FCL to the control system.

\section{Introduction}

The principle of Series resonant fault current limiter(SRFCL) is based on that reactance and capacitance in the resonant frequency. The speed of bypassing capacitor determines limiter speed. Short circuit fault identification is the key to fast trigger strategy ${ }^{[1-7]}$. Current wave through the series reactor capacity is very weak, resulting in traveling wave protection reliability is not high ${ }^{[9]}$. The restrictor body signal is easy to measure, with good response characteristics. It is very necessary to study the signal in the basement of body criterion .

Literature [10-11] introduces short current instantaneous value and current differential rate method .Literature [12] studies the superconducting current limiter quick trigger method. . Literature [13] studies the quick identification of short current circuit. The principle is based on that phase shifter produces phase lag and the level fault signal mutation mechanism. This paper presents method to quickly identify short current. The method uses resonant components normal voltage difference is 0 , the resonant component voltage difference after fault will mutate. The method affected by closing the distance and angle is small, fast fault detection and effective action will reduce limiter current and voltage stress.

\section{$\underline{\text { Resonant voltage difference identification method }}$}

\section{A. Recognition principle}

SRFCL Quick Starting affect devices working conditions, the capacitor over-voltage protection device zinc oxide arrester (MOV) energy consumption increases linearly with time. The longer action, MOV greater energy consumption, over-voltage arrester as the main protection devices will choose high-capacity configuration. MOV time longest in SRFCL electrical transient variation,. short current is most direct criterion. Resonant voltage difference is fast mutation signal. The paper analyzes the signal mutation characteristics of resonant voltage difference.

During the steady state, limiting element resonant at system frequency, current-limiting reactor voltage and resonant capacitor voltage equal in magnitude and opposite in direction, so the scalar difference is 0 . Set time for failure at $t_{0}$, fault duration is $t_{\text {fault }}$. MOV action time is $t_{1}$. In the interval $\left[t_{0}, t_{1}\right]$, according to short additional state superposition method, the fault current is: 


$$
\begin{aligned}
i(t)= & I_{m} \operatorname{Sin}(\omega t+\alpha-\varphi)+I_{m 1} \operatorname{Sin}\left(\omega t+\varphi_{i}\right) \\
& +I_{m 2} \sqrt{\operatorname{Cos}^{2} \varphi_{d}+\left(\frac{\omega_{f}}{\omega}\right)^{2} \operatorname{Sin}^{2} \varphi_{d} \operatorname{Cos}\left(\omega_{p} t+\theta\right) e^{-\beta_{p} t}}
\end{aligned}
$$

$\varphi_{i}$ is the fault impedance angle, $\omega$ is system frequency, $\omega_{0}$ is system resonant frequency, $\beta_{\mathrm{p}}$ is the damping coefficient, $\theta$ is the resonant current impedance angle, $\varphi_{d}$ is phase angle for the short initial time, $I_{m 1}$ is the current limiter into the system short-circuit steady-state, $I_{m 2}$ is current for oscillation attenuation current. From the equation, current limiter with capacitive branch result in short current decay in the cosine component. The current rate of change and the resonant voltage difference:

$$
\begin{aligned}
& \frac{d i}{d t}=\omega I_{m} \operatorname{Cos}(\omega t+\alpha-\varphi)+\omega I_{m 1} \operatorname{Cos}\left(\omega_{t}+\varphi_{i}\right) \\
& -I_{m 2} \sqrt{\operatorname{Cos}^{2} \varphi_{d}+\left(\frac{\omega_{p}}{\omega}\right)^{2} \operatorname{Sin}^{2} \varphi_{d}} * e^{-\beta_{p} t}\left(\omega_{p} \operatorname{Sin}\left(\omega_{p} t+\theta\right)+\beta_{p} \operatorname{Cos}\left(\omega_{p} t+\theta\right)\right) \\
& \Delta u=-I_{m 2} \sqrt{\operatorname{Cos}^{2} \varphi_{d}+\left(\frac{\omega_{p}}{\omega}\right)^{2} \operatorname{Sin}^{2} \varphi_{d} *} \\
& e^{-\beta_{t} t}\left(\left(\omega_{0} L+\frac{1}{c} * \frac{\beta_{p}}{\omega_{p}^{2}+\beta_{p}^{2}}\right) \operatorname{Sin}\left(\omega_{p} t+\theta\right)+\left(\beta_{p} L+\frac{1}{c} * \frac{\omega_{p}}{\omega_{0}^{2}+\beta_{p}^{2}}\right) \operatorname{Cos}\left(\omega_{p} t+\theta\right)\right)
\end{aligned}
$$

$L$ is current-limiting reactor, $C$ is the resonant capacitor. Equation (2) concludes that the current rate mutation depends on $\omega I_{m 1}$, the resonant voltage difference depends on $I_{m 2}$. Figure 1 is resonant voltage curve at fault time. Fault time at $200 \mathrm{~ms}$, reactance voltage can be considered equivalent to the current rate of change. Current rate and the resonant voltage difference are significantly at initial time of the short current transition, the resonant voltage difference due to the initial moment is 0 , the amplitude of transition is more intense.

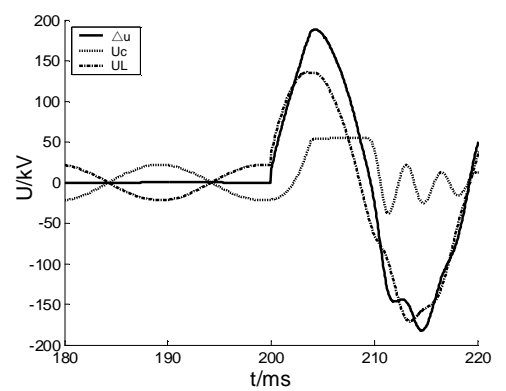

Figure 1. The curve of Resonant Component Voltage

\section{B. Time characteristics}

In the background of the East China Power Grid 500kV current limiter project, the original short-circuit current up to $51 \mathrm{kA}$, after accessing limiting device, the current-limiting reactor $8 \Omega$, the short current is controlled under $47 \mathrm{kA}$.

Protection criterion threshold: current instantaneous value is $8 \mathrm{kA}$, the current rate of change is $2 \mathrm{~A} / \mathrm{us}$, resonant voltage difference is $15 \mathrm{kV}$. Point of failure is set to $5 \%, 35 \%, 55 \%, 75 \%$ of the fault line, closing angle from 0 degrees to 180 degrees, fault tape for the three-phase ground, two-phase short, two-phase, the single phase. Lots of simulation is to do research on response time characteristics of the protection criterion, three-phase ground time curve shown in Figure 2. 


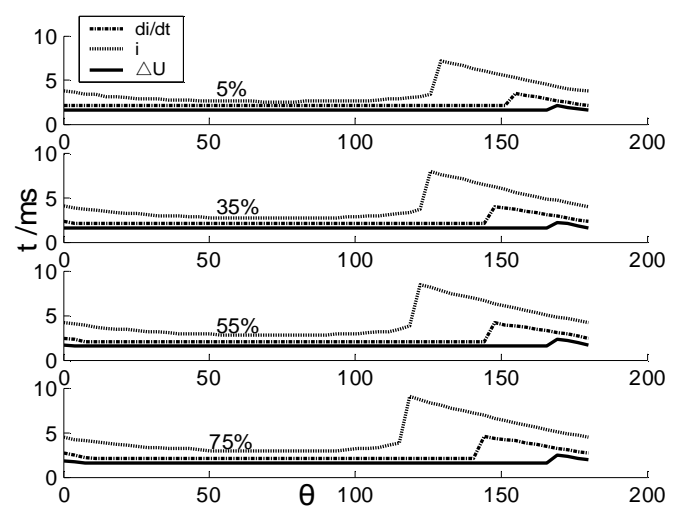

Fig. 2 The curve of Protection Criterion Time

Line exit fault of the three-phase ground show that the voltage difference time is $2.2 \mathrm{~ms}$, the current rate time is $2.8 \mathrm{~ms}$. In the closing angle of 160 degrees, the current rate time jump into $4 \mathrm{~ms}$, the resonant voltage difference jump into $3 \mathrm{~ms}$. Simulation results show that in different locations and different fault closing angle, the resonant voltage difference criterion action time is always the fastest, the current rate is faster, instantaneous current is slowest.

Short current method affected by many factors, selecting the threshold to avoid a lot of interference. current rate has more higher sensitivity than instantaneous current. But there are two confounding factors: First, as the power supply system has most inductive load, which cause the current rate of change of mutation. Second, the impact on the system power supply system in the operation of larger movements, such as motor starting, large equipment starting and stopping, frequency control, the system operation.

\section{protection criterion scheme}

\section{Opening and closing of circuit breaker}

When line breaker open and reclose, resonant voltage difference signal mutate and malfunction signal will be sent, it is shown in Figure 3. Breaker open at $280 \mathrm{~ms}$, after a half cycle, current is zero. Voltage difference mutate within $3 \mathrm{~ms}$ after breaker open, after half cycle, the resonant voltage equals the port of capacitor voltage.

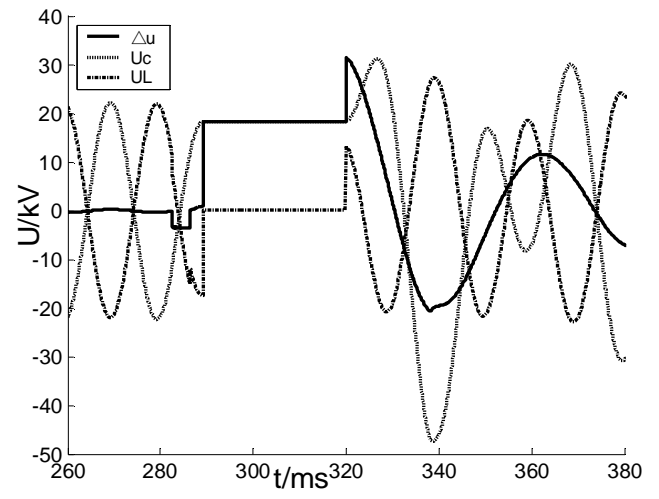

Fig.3 The curve of Resonant Component Voltage

when Breaker Close or Open

During reclosing process resonant voltage difference will decay over time at end of resonant device. Simulation indicates that this value is effected by closing angle. Are selected closing angle $0^{\circ}$, $60^{\circ}, 90^{\circ}, 150^{\circ}$ to do simulation analysis. Simulation results show that in the reclosing process, resonant voltage difference is changed with time. 


\section{Logical relationship}

It is simple for resonant voltage difference criterion to identify fault not to escape the system breaking fault. Resonant voltage difference signal coordinated with the current instantaneous value can be identified as a criterion.

After reclosing, the circuit into the steady-state, until the voltage difference decay to below the threshold criterion of action, and then open the device to ensure that the limiting control device will not malfunction in the closing moments.

During normal operation, the resonant voltage difference and current criterion results through logic gate circuit constructs line broken failure. When the device to determine system fault occurs, the signal triggers limiter thyristor. The results of $I>I_{\text {set }}$ through the inverter and the resonant voltage difference to determine the results through logic gate constitutes disconnection fault. Value of $T_{\text {chzys }}$ can be got under various operating systems and left some safety margin.

Hardware circuit is shown in Figure 4 (b). AD1674 is high-speed sampling chip, parallel 12, 128 sampling points per cycle sampling, the sampling rate of 12.8k / s. VQG100FPGA using Actel's FPGA chip, the chip has a size of 60K system gates, 18k SRAM, 71 I / O. FPGA implementation logic, and provides control AD signals and alarm signal.

Experimental threshold selection: the current threshold of $150 \mathrm{~mA}$, threshold current change rate $34 \mathrm{~A} / \mathrm{s}$, the resonant voltage difference threshold is $6.8 \mathrm{~V}$. Typical fault recorder waveform is shown figure4 (a) below. Jumping voltage level signal is resonant voltage difference signal criterion, the curve for the resonant voltage difference signal. Done for short fault 20 groups randomly, criterion export time data is shown in Figure 4(b) below. Statistical time results show that the resonant voltage difference response time is $0.6 \mathrm{~ms}$, the current rate response time is $2 \mathrm{~ms}$, short-circuit current response time is $6 \mathrm{~ms}$.

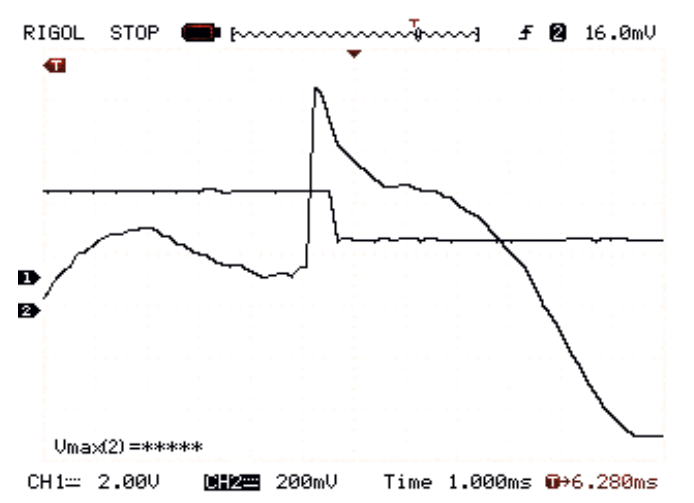

(a) The curve of resonant voltage difference

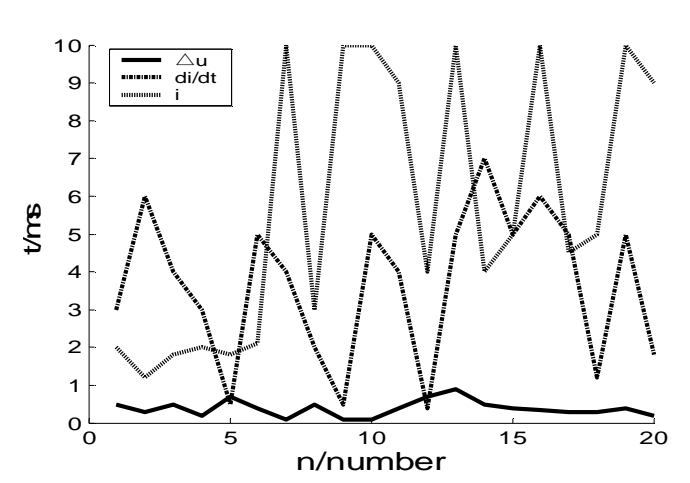

( b ) Statistical Time

Fig.4 Experimental Data and Waveform

\section{Conclusion}

Resonant voltage difference is the resonant element mutation. The method mutate more significantly than the current rate, effected less by the phase angle and fault distance; It is influenced by the circuit breakers open and close, with the short current amplitude criterion and the combination of re-closing, interference will be avoided for line open and close; Model experiments show that the resonant voltage difference identification method can provide criteria for the export signal in $1 \mathrm{~ms}$.

\section{Reference}

[1] Sun Qizhen, Cai Zexiang, Li Aimin. A Short-circuit Current Over-limited Mechanism of $500 \mathrm{kV}$ Power System and the Adaptability of Limiting Measures[J]. Automation of electric Power Systems, 2009,33(21):92-96. 
[2] J. Amon F., P. C. Fernandez, E. H. Rose, A. D'Ajuz, A. Castanheira , Brazilian Successful Experience in the Usage of Current Limiting Reactors for Short-Circuit Limitation, power systems transients( IPST' 05) IN Montreal, Canada on June 19-23,2005.

[3] Cigre Working Group A3.10. Fault Current Limiters in Electrical Medium and High Voltage Systems. Paris: Cigre, 2003.

[4] Hargava, Bharat, Haas, Richard G. Thyristor protected series capacitors project at Southern California Edison Co. Proceedings of the IEEE Power Engineering Society Transmission and Distribution Conference, 2002:1493-1498.

[5] Nagata. M, Tanaka. K, et al, FCL Location Selection in Large Scale Power System, IEEE Transactions on applied superconductivity, Vol.11 , NO. 1, March 2001, pp. 2489 2495.

[6] Huaxin Wang, Hexun Xi, Guangfu Tang, Jianchao Zhen. Research of Main Circuit on the Series Resonance Fault Current Limiter. The 5th International Conference on Power System Technology, Powercon2006, Chongqing, China, 22-26 October 2006.

[7] WANG Hua-xin, WANG Hua-feng, TANG Guang-fu, ZHEN Jian-cao . The Design and Analysis on Overvoltage Protection Circuit Topology Structure[J]. Proceedings of the CSEE, 2008, 28(15):1-6.

[8] GAO Jing, ZHENG Jianyong, PAN Zhendong. Study of improved Fourier algorithm for microprocesson-based protection in power system[J]. Relay, 2002,30(10) : 16-20. (in Chinese).

[9] Ge Yaozhong, Dong Xing, Dong Xinzhou, Suonan Jiale. A New Bus-Bar Protection Based on Current Travelling Waves and Wavelet Transform-Basic Principle and Criterion[J]. Transactions of China Electrotechnical Society, ,2003,18(2):95-100.

[10] Chen Gesong, Lin Jimin, Guo Jianbo. Overvoltage Protection For $500 \mathrm{kV}$ Series Compensation Station [J]. Power System technology, 2001, 25(2) : 21-26(in Chinese).

[11] Jin Xue-fen, Dai Chao-bo, Wu Shou-yuan, Jing Ping. Influence of Fault Current Limiter on Protective Relaying[J]. Power System technology, 2009,33(9):29-33. (in Chinese).

[12] He Yi, Wu Aiguo, Xin Ying. Rapid Pattern Recognition of Fault Current for HTS Three-Phase Saturated Iron Core Fault Current Limiter[J]. Transactions of China Electrotechnical Society, 2009, 24(1):81-88.

[13] Zheng Qingshui, Ma Zhiying, Qian Hua. Research on a Fast Identified Circuit of Short-circuit Current[J]. Transactions of China Electrotechnical Society, , 2004, 19(3):99-103. 